\title{
Characterization of a midgut mucin-like glycoconjugate of Lutzomyia longipalpis with a potential role in Leishmania attachment
}

\author{
Jitka Myšková1*, Anna Dostálová1, Lucie Pěničková1, Petr Halada², Paul A. Bates ${ }^{3}$ and Petr Volf ${ }^{1}$
}

\begin{abstract}
Background: Leishmania parasites are transmitted by phlebotomine sand flies and a crucial step in their life-cycle is the binding to the sand fly midgut. Laboratory studies on sand fly competence to Leishmania parasites suggest that the sand flies fall into two groups: several species are termed "specific/restricted" vectors that support the development of one Leishmania species only, while the others belong to so-called "permissive" vectors susceptible to a wide range of Leishmania species. In a previous study we revealed a correlation between specificity vs permissivity of the vector and glycosylation of its midgut proteins. Lutzomyia longipalpis and other four permissive species tested possessed O-linked glycoproteins whereas none were detected in three specific vectors examined.
\end{abstract}

Results: We used a combination of biochemical, molecular and parasitological approaches to characterize biochemical and biological properties of O-linked glycoprotein of Lu. longipalpis. Lectin blotting and mass spectrometry revealed that this molecule with an apparent molecular weight about 45-50 kDa corresponds to a putative $19 \mathrm{kDa}$ protein with unknown function detected in a midgut CDNA library of Lu. longipalpis. We produced a recombinant glycoprotein rLuloG with molecular weight around $45 \mathrm{kDa}$. Anti-rLuloG antibodies localize the native glycoprotein on epithelial midgut surface of Lu. longipalpis. Although we could not prove involvement of LuloG in Leishmania attachment by blocking the native protein with anti-rLuloG during sand fly infections, we demonstrated strong binding of rLuloG to whole surface of Leishmania promastigotes.

Conclusions: We characterized a novel O-glycoprotein from sand fly Lutzomyia longipalpis. It has mucin-like properties and is localized on the luminal side of the midgut epithelium. Recombinant form of the protein binds to Leishmania parasites in vitro. We propose a role of this molecule in Leishmania attachment to sand fly midgut.

Keywords: Phlebotomine sand flies, Leishmania, Lipophosphoglycan, Glycoprotein

\section{Background}

Leishmania protozoans, the causative agents of leishmaniases, are transmitted by bites of female phlebotomine sand flies. In the sand fly vector, Leishmania parasites must overcome various barriers to generate transmissible infections and ensure continuation of the

\footnotetext{
* Correspondence: jpeck@seznam.cz

'Department of Parasitology, Faculty of Science, Charles University, Viničná 7, 12844 Prague 2, Czech Republic

Full list of author information is available at the end of the article
}

life-cycle (reviewed by [1]). A crucial step in the vector phase of the Leishmania life-cycle is the binding of promastigotes to the sand fly midgut. Promastigotes insert their flagella into the microvillar border of the midgut epithelium and anchor themselves to the midgut surface $[2,3]$. This attachment enables them to persist in the sand fly gut when the blood meal remnants are defecated.

A series of studies on the parasite-vector combination Leishmania major-Phlebotomus papatasi established an 
important paradigm; galactose residues on monogalactosylated phosphodisaccharide repeats of the major surface glycoconjugate lipophosphoglycan (LPG) have been shown to bind to a galectin located on the surface of the sand fly midgut [4-7]. However, a more recent study reported partial involvement of the flagellar protein FLAG1/SMP1 in attachment of $L$. major to the midgut epithelium of P. papatasi [8], and earlier experiments with LPG-deficient mutants revealed that LPG is not required for the Leishmania attachment in many other sand fly species [9-11]. These studies indicate that alternative attachment molecules can be involved in midgut binding in addition to LPG-galectin.

Laboratory studies on vector competence to Leishmania parasites suggest that sand flies fall into two broad groups. Three species, i.e. P. papatasi, P. duboscqi and P. sergenti, are termed "specific" or "restricted" vectors that support the development of one Leishmania species only (L. major and L. tropica, respectively). Other sand fly species, importantly including vectors of the visceralising parasites L. donovani and L. infantum, belong to socalled "permissive" vectors, which are susceptible for a wide range of Leishmania spp. In laboratory conditions Leishmania parasites are capable of developing in any permissive vector, if given the opportunity (reviewed by $[1,12]$ ).

In a study exploring the molecular basis of vector competence Myšková and colleagues [9] revealed a remarkable correlation between specificity $v s$ permissivity of the vector and the glycosylation of its midgut proteins. The Helix pomatia agglutinin (HPA), a lectin specific for terminal $\mathrm{N}$-acetyl-galactosamine (GalNAc) present on O-linked glycoconjugates, bound to midgut proteins from permissive but not from specific vectors. All five permissive species tested possessed HPA positive bands, whereas none were detected in the three specific vectors examined $[9,10,13]$. These findings suggested a hypothesis for the role of O-linked glycoconjugates in Leishmania binding, which was supported by two further observations. Fluorescent-labelled HPA (FITCHPA) showed specific localisation of O-glycosylated epitopes in the microvillar border of sand fly midgut, the location required for Leishmania attachment, and the O-linked glycoconjugates recognised by FITC-HPA bound to the surface of Leishmania promastigotes [9].

In the present work we describe an O-linked glycoconjugate of the permissive vector $L u$. longipalpis and show that it has mucin-like properties. Functional testing was performed using the recombinant form of the $L u$. longipalpis glycoconjugate (rLuloG) and specific anti-rLuloG antibodies using in vivo and in vitro methods. For in vivo studies, experimental infections of $\mathrm{Lu}$. longipalpis with $L$. infantum were performed as described previously for L. major and P. papatasi galectin [6]: L. infantum parasites were mixed with blood reconstituted with specific anti- rLuloG serum to test for blocking of parasite development in the sand fly midgut. In vitro experiments were performed to investigate the binding of rLuloG to Leishmania parasites.

\section{Methods}

\section{Sand fly colonies and parasites}

Laboratory colonies of two sand fly species were used: Lutzomyia longipalpis (origin from Jacobina, Brazil) and Phlebotomus papatasi (origin from Turkey). Colonies were maintained in conditions described previously [14] and fed on $50 \%$ sucrose. Most experiments were done with 4-7 day-old females, and the expression of LuloG was compared in females aged from 1 to 12 days.

The following parasite isolates were used: Leishmania infantum (MHOM/BR/76/M4192) and Leishmania major LV561 (MHOM/IL/67/LRC-L137). Parasites were maintained at $26{ }^{\circ} \mathrm{C}$ on Medium 199 supplemented with $20 \%$ foetal bovine serum (Thermo Fisher Scientific, Waltham, USA). Prior to sand fly infections, Leishmania parasites were washed by centrifugation and resuspended in saline.

\section{Detection of O-linked glycoconjugates in Lutzomyia longipalpis midguts}

Female midguts were homogenized mechanically by repeated freezing and thawing, proteins were separated by SDS-PAGE (10\% gel, reducing conditions, 10 guts per lane), transferred to nitrocellulose and analyzed by lectin blotting. The nitrocellulose membrane was incubated with Tris-NaCl-Tween (20 mM Tris, $150 \mathrm{mM} \mathrm{NaCl}$, $\mathrm{pH}$ 7.6) and blocked with $5 \%(\mathrm{w} / \mathrm{v})$ bovine serum albumin (BSA; Sigma-Aldrich, St. Louis, USA; diluted in Tris- $\mathrm{NaCl}$-Tween) overnight at $4{ }^{\circ} \mathrm{C}$ and then for $1 \mathrm{~h}$ at room temperature with biotinylated Helix pomatia agglutinin (HPA; $1 \mu \mathrm{g} / \mathrm{ml}$; Sigma-Aldrich). After repeated washing the blots were incubated with streptavidin peroxidase $(2.5 \mu \mathrm{g} / \mathrm{ml}$; Sigma-Aldrich, St. Louis, USA; in Tris- NaCl-Tween) and the peroxidase reaction was developed in 4-chloro-1-naphthol solution (SigmaAldrich). Experiments were done with females of various ages and physiological states (e.g. females which did not feed on blood, females after blood-feeding) as indicated.

\section{Characterization of the midgut glycoconjugate}

Lysates were prepared from 20 midguts of Lu. longipalpis females. Triton X-114 was used for separation of hydrophilic and amphiphilic proteins as previously decribed by Bordier [15] with following modifications: the lysates were incubated with the $1 \%$ Triton X-114 for $90 \mathrm{~min}$ on ice and the separation was done without sucrose cushion. Triton $\mathrm{X}-114$ is a non-ionic detergent with a low cloud point (about $20-23{ }^{\circ} \mathrm{C}$ ) enabling 
protein solubilization with phase-partitioning of hydrophilic from amphiphilic proteins. The aqueous and the detergent phase were then analyzed by SDS-PAGE and blotting with HPA (as decribed above). To study the presence of a glycophosphatidylinositol (GPI) anchor, lysates in Tris- $\mathrm{NaCl}$ were incubated for $1.5 \mathrm{~h}$ at $37^{\circ} \mathrm{C}$ with protease inhibitors (Complete, Roche, Mannheim, Germany) and 1 unit phosphatidylinositol-specific phospholipase $\mathrm{C}$ (PI-PLC), a highly purified enzyme prepared from Bacillus cereus (Sigma-Aldrich). The sample was then fractionated with Triton X-114 and analyzed by SDS-PAGE followed by blotting with HPA.

To check the isoelectric point of the candidate glycoconjugate, a lysate of 50 midguts was analyzed by 2D electrophoresis (IEF/SDS-PAGE), followed by blotting with HPA incubation as described above.

To generate sufficient material for protein identification, HPA affinity chromatography was used to purify the Olinked glycoconjugate from midguts of $\mathrm{Lu}$. longipalpis females. Lysates of 1400 midguts were incubated with 9 units of PI-PLC in Tris- $\mathrm{NaCl}$ and protease inhibitors (Complete, Roche) for $1.5 \mathrm{~h}$ at $37{ }^{\circ} \mathrm{C}$ and loaded onto a HPA-agarose column (Sigma-Aldrich). After washing, specifically bound molecules were eluted with $0.5 \mathrm{M}$ GalNAc in Tris- $\mathrm{NaCl}$ and analyzed by SDS-PAGE (10 \% gel, reducing conditions) or 2D electrophoresis (IEF/SDS-PAGE). The glycoconjugate band/spot was stained by Pro-Q-Emerald glycoprotein stain (Thermo Fisher Scientific), a technology available for detection of glycoconjugates in gels. The protein band/spot was excised from the gel, cut into small pieces and washed using $50 \mathrm{mM}$ 4-ethylmorpholine acetate (pH 8.1) in $50 \%$ acetonitrile $(\mathrm{MeCN})$. The protein was reduced with $30 \mathrm{mM}$ Tris(2-carboxyethyl)phosphine (TCEP) in $100 \mathrm{mM}$ Tris$\mathrm{HCl}(\mathrm{pH} 8.0)$ at $65{ }^{\circ} \mathrm{C}$ for $30 \mathrm{~min}$ and alkylated by $30 \mathrm{mM}$ iodacetamide in $100 \mathrm{mM}$ Tris- $\mathrm{HCl}(\mathrm{pH} 8.0)$ for $60 \mathrm{~min}$ in the dark. The gel pieces were then incubated overnight at $37{ }^{\circ} \mathrm{C}$ in a cleavage buffer containing $25 \mathrm{mM}$ 4ethylmorpholine acetate, $5 \% \mathrm{MeCN}$ and trypsin (100 ng; Promega, Woods, USA) or Asp-N protease (30 ng; Roche). The resulting peptides were extracted into $40 \% \mathrm{MeCN} /$ $0.1 \%$ TFA. One $\mu \mathrm{l}$ of the peptide mixture was deposited on a MALDI plate, allowed to air-dry at room temperature and overlaid with $0.4 \mu \mathrm{l}$ of MALDI matrix (aqueous $50 \%$ MeCN/0.1 \% TFA solution of $\alpha$-cyano-4-hydroxycinnamic acid; $5 \mathrm{mg} / \mathrm{ml}$; Sigma-Aldrich). Mass spectra were measured on an Ultraflex III MALDI-TOF instrument (Bruker Daltonics, Bremen, Germany) equipped with LIFT technology for MS/MS analysis. MS data were searched using an in-house MASCOT search engine against the NCBI nr database subset of eukaryotic proteins.

\section{Expression and purification of rLuloG in $\mathrm{H} 5$ insect cells} RNA was isolated from 10 midguts of Lutzomyia longipalpis females using a High Pure RNA isolation kit
(Roche) and reverse-transcribed using Superscript III reverse transcriptase (Thermo Fisher Scientific) following the manufacturer's instructions. The sequence of the cDNA corresponding to transcript GenBank: EU124597 was amplified using Q5 High Fidelity DNA polymerase (BioLabs, Ipswich, USA) (forward primer: 5' -CTT AAA TGC TAC AAT TGC AAT TCC T-3' and reverse primer: 5'-ACT ACT CTC AGT TGT AGT TGG A-3') omitting the predicted signal peptide (SignalP, Lyngby, Denmark) and GPI anchoring signal (PredGPI, gpcr2.biocomp.unibo.it). The resulting amplicon was cloned into pFastBac HBM TOPO vector and expressed in High Five Trichoplusia ni cells using the Bac-to-Bac HBM TOPO secreted expression system (Thermo Fisher Scientific) following the manufacturer's instructions. Infected High Five cells were scraped from Petri dishes and transfered to a microcentrifuge tube. The solution was centrifuged first at $1500 \mathrm{rpm}$ for $7 \mathrm{~min}$ and the supernatant was concentrated using $30 \mathrm{kDa}$ filters Amicon Ultra-15 (Millipore, Carrigtwohill, Ireland), according to the manufacturer's instructions. The resulting sample was incubated with HPA agarose (Sigma-Aldrich) for $2 \mathrm{~h}$. The recombinant protein named rLuloG was specifically eluted with $0.5 \mathrm{M}$ GalNAc in $\mathrm{Tris}-\mathrm{NaCl}$ and washed several times in Tris- $\mathrm{NaCl}$ on $30 \mathrm{kDa}$ filters. The purity and molecular weight was confirmed by SDS-PAGE (10 \% gel, reducing conditions) followed by Coomassie blue staining and blotting with HPA (as described above). The band was excised from the gel, digested by trypsin and analyzed by MALDI-TOF mass spectrometry as described above.

\section{Rabbit anti-rLuloG antibodies: western blotting and} indirect immunofluorescence with Lu. longipalpis midguts Polyclonal anti-rLuloG antibodies were commercially prepared by repeated immunization of rabbit with $2 \mathrm{mg}$ of purified rLuloG (Eurogentec, Seraing, Belgium). Antibody binding to rLuloG and to native protein from Lu. longipalpis midguts (10 guts per lane) was analyzed by SDS-PAGE (10\% gel, reducing conditions) followed by western blotting. The nitrocellulose membrane was incubated with Tris-NaCl-Tween $(20 \mathrm{mM}$ Tris, $150 \mathrm{mM} \mathrm{NaCl}, \mathrm{pH}$ 7.6) and blocked with $6 \%$ milk diluted in PBS/Tween overnight at $4{ }^{\circ} \mathrm{C}$. The membrane washed with PBS/Tween was incubated first for $1 \mathrm{~h}$ with anti-rLuloG serum (1: 15,000 in $3 \%$ BSA in PBS/Tween), repeatedly washed and then incubated with peroxidase-conjugated anti rabbit IgG SwAR/ Px (Sevapharma a.s., Prague, Czech Republic), 1:1000 in 3 \% BSA in PBS/Tween, as the secondary antibodies. After repeated washing the colour reaction was developed in 4chloro-1-naphthol solution. Preimmune rabbit sera or midguts of the specific vector $P$. papatasi were used as negative controls. Incubation of separated rLuloG with HPA and inhibiton of this reaction with specific GalNAc were also tested. 
Anti-rLuloG antibodies were also used to localize LuloG in sand fly midguts. To mimic the natural situation during Leishmania infections, midguts were dissected from Lu. longipalpis females which had defecated blood meal remnants (4-6 days post-blood meal). Midguts were longitudinally opened, fixed in $2 \%$ paraformaldehyde for $20 \mathrm{~min}$, washed twice in Grace's Insect Medium (GIM, Sigma-Aldrich) and incubated with preimunne serum or anti-rLuloG antibodies (1:300 in $1 \%$ BSA in GIM) for $1 \mathrm{~h}$ in RT. Guts were then washed four times in GIM and incubated with FITC antirabbit IgG (1: 50 in $1 \%$ BSA in GIM) for $1 \mathrm{~h}$. After washing in GIM, guts were placed in $2 \mu \mathrm{l}$ of cooled CyGel (Biostatus Limited, Leicestershire, UK) on a microscope slide, carefully flattened, mounted in Prolong Gold antifade reagent (Thermo Fisher Scientific) and examined under a fluorescent microscope Olympus BX51.

\section{Binding of rLuloG to Leishmania promastigotes}

A 3-day culture of L. major LV561 was washed by centrifugation with PBS, fixed with $2 \%$ formaldehyde for $30 \mathrm{~min}$, washed again, diluted to a final density of $10^{7}$ promastigotes $/ \mathrm{ml}$, spotted onto a slide and air-dried. Fixed parasite cells were rehydrated in PBS for $5 \mathrm{~min}$, blocked with $0.1 \%$ BSA in PBS for 5 min and incubated with rLuloG for $1 \mathrm{~h}$. Slides incubated with preimunne serum or $0.1 \%$ BSA served as negative controls. After washing (PBS for $15 \mathrm{~min}$ ) the cells were incubated first with anti-rLuloG serum (1:500 in 0.1 \% BSA in PBS) for $1 \mathrm{~h}$, washed and then incubated with ALEXA fluor 488 goat anti-rabbit IgG (Thermo Fisher Scientific; 1:500 in $0.1 \%$ BSA in PBS) in the dark. Slides were dried and mounted in Prolong Gold antifade mounting medium (Thermo Fisher Scientific) and examined under the fluorescent microscope Olympus BX51.

\section{Leishmania development in Lu. longipalpis: effect of anti-rLuloG serum}

Sand fly females were infected by feeding through a chick skin membrane with $L$. infantum promastigotes from 3-day-old cultures (cell density of $5 \times 10^{5} / \mathrm{ml}$ ) in heat-inactivated rabbit blood (Bioveta, Ivanovice na Hané, Czech Republic) reconstituted either with antirLuloG serum or control preimmune serum. Bloodengorged females were separated and maintained at $26^{\circ} \mathrm{C}$. On day 2 post-blood meal (PBM) (i.e. before defecation) or day 4 and 7 PBM (i.e. after defecation of blood meal remnants) the females were dissected for microscopical examination.

Parasite density in dissected midguts was graded according to criteria reported previously as light $(<100$ parasites/gut), moderate (100-1000 parasites/gut) or heavy (> 1000 parasites/gut) [16]. Experiments were repeated three times. Infection rates (number of infected $v s$ uninfected females) and intensities of infection (heavy, moderate, light, zero) were compared between groups using Chi-square test and S-PLUS 2000 programme.

\section{Results}

Detection and characterization of midgut glycoconjugate Midgut lysates of the permissive vector Lu. longipalpis were analyzed by SDS-PAGE, followed by blotting with Helix pomatia agglutinin. This lectin is specific for GalNAc, a carbohydrate typically associated with O-linked glycan-displaying glycoconjugates such as mucins. HPA showed reactivity with a molecule of around $45-50 \mathrm{kDa}$ molecular mass; under reducing SDS-PAGE we also detected one additional band around $35 \mathrm{kDa}$. These sand fly O-linked glycoconjugates do not resolve into sharp bands under SDS-PAGE as commonly found for heavily glycosylated proteins, and are relatively acidic with $\mathrm{pI}$ values from 3 to 5 , as indicated by IEF/SDS-PAGE (Fig. 1). The glycoconjugate was detectable during the whole life-cycle of adult females, i.e. after hatching, before and after blood-feeding (Fig. 2).

Extraction of sand fly midgut membranes with Triton X-114 before PI-PLC treatment recovered the HPA binding molecule in the detergent-rich phase, whereas after PI-PLC treatment most of the glyconconjugate was present in the aqueous phase (Fig. 3). These results are clearly indicative of a GPI membrane anchor for the glycoconjugate.

The PI-PLC-treated sample was subjected to HPAaffinity chromatography and the eluted fraction separated by SDS-PAGE. Mass spectrometry of the

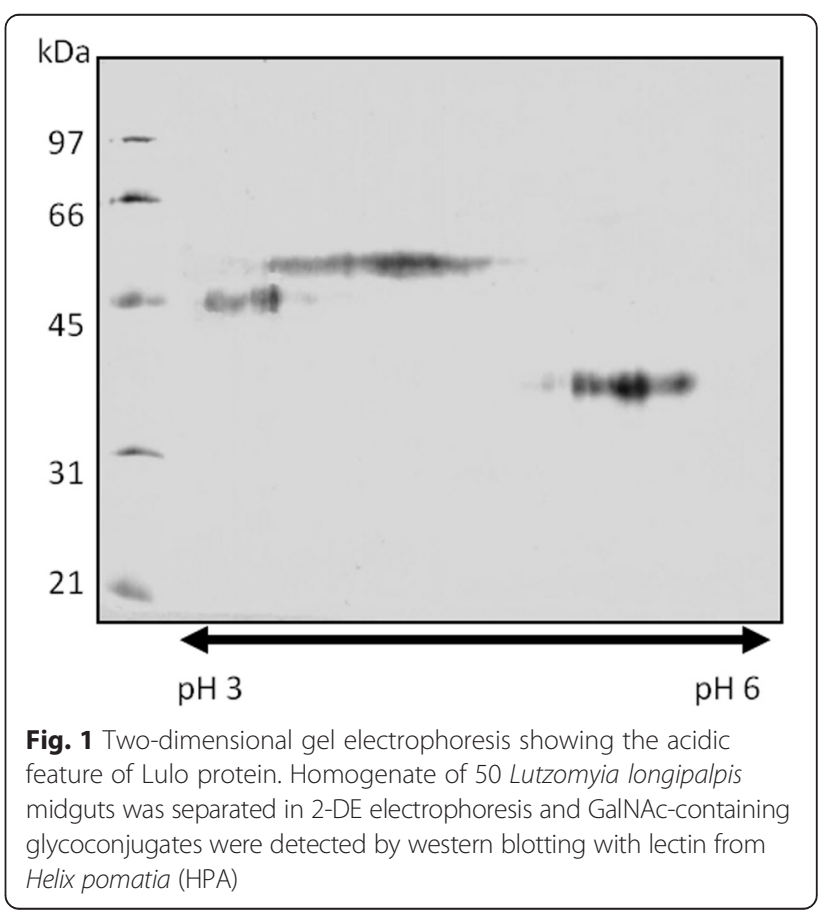




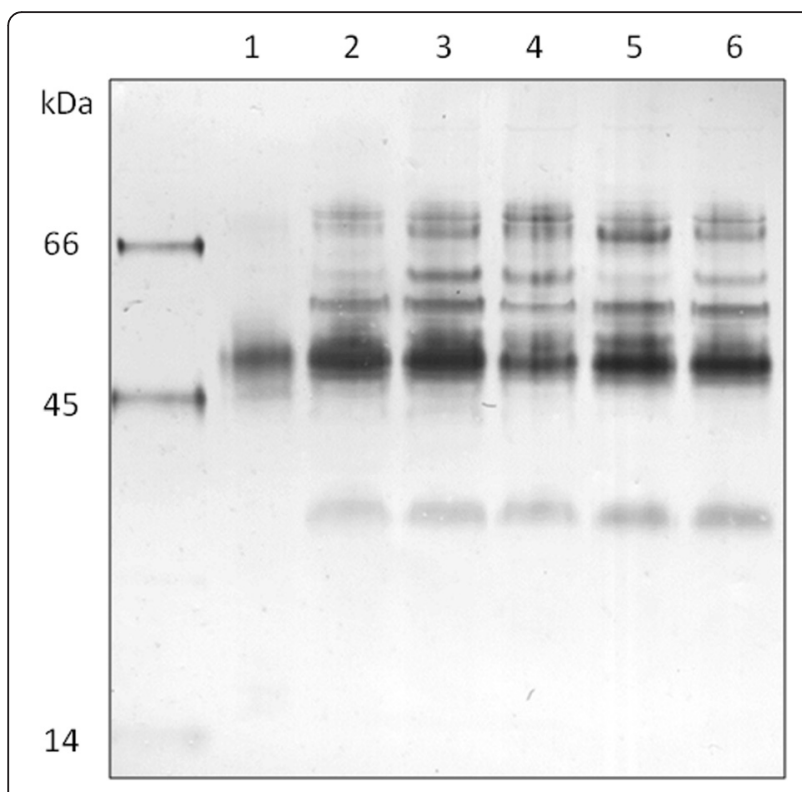

Fig. 2 Detection of the GalNAc-containing glycoconjugate during the life of adult females. Homogenate of ten Lu. longipalpis midgut was separated by SDS-PAGE followed by western blot with lectin from Helix pomatia (HPA). Lane 1: 1-day-old females; Lane 2: 4-dayold females; Lane 3: 8-day-old females; Lane 4: 8-day-old females after blood meal; Lane 5: 12-day-old females; Lane 6: 12-day-old females after blood meal

glycoconjugate spot identified peptides resulting from both trypsin- and AspN-digestion (YGQATVEGQEITLR and DLIVGQHIFY, respectively) corresponding to a Lutzomyia longipalpis $19 \mathrm{kDa}$ midgut protein (GenBank: ABV60315). The C-terminal part of the protein sequence is rich in potentially O-glycosylated Ser/Thr residues (NetOGlyc prediction), forming a mucin-like domain (Fig. 4). This is in agreement with the observed lectin-binding properties of this glycoconjugate. Moreover, the high number of carbohydrate residues likely account for the majority of the apparent molecular weight of the molecule.

\section{rLuloG, anti-rLuloG and their binding to Leishmania promastigotes and sand fly midguts}

Constrained by the small amount of the native molecule available, we opted for producing a recombinant version, in order to be able to produce antibodies specifically recognizing the identified glycoconjugate. We used the baculovirus secreted expression system in High Five insect cells, which allows for expression of glycosylated proteins. The purified recombinant rLuloG was subjected to SDS-PAGE, followed by western blotting and compared to the native mucin-like molecule (Fig. 5). rLuloG had a molecular weight around $40-45 \mathrm{kDa}$ and did not resolve into sharp bands, similar to the native midgut protein. The identity of rLuloG was confirmed by MALDI-TOF mass spectrometry yielding 13 tryptic

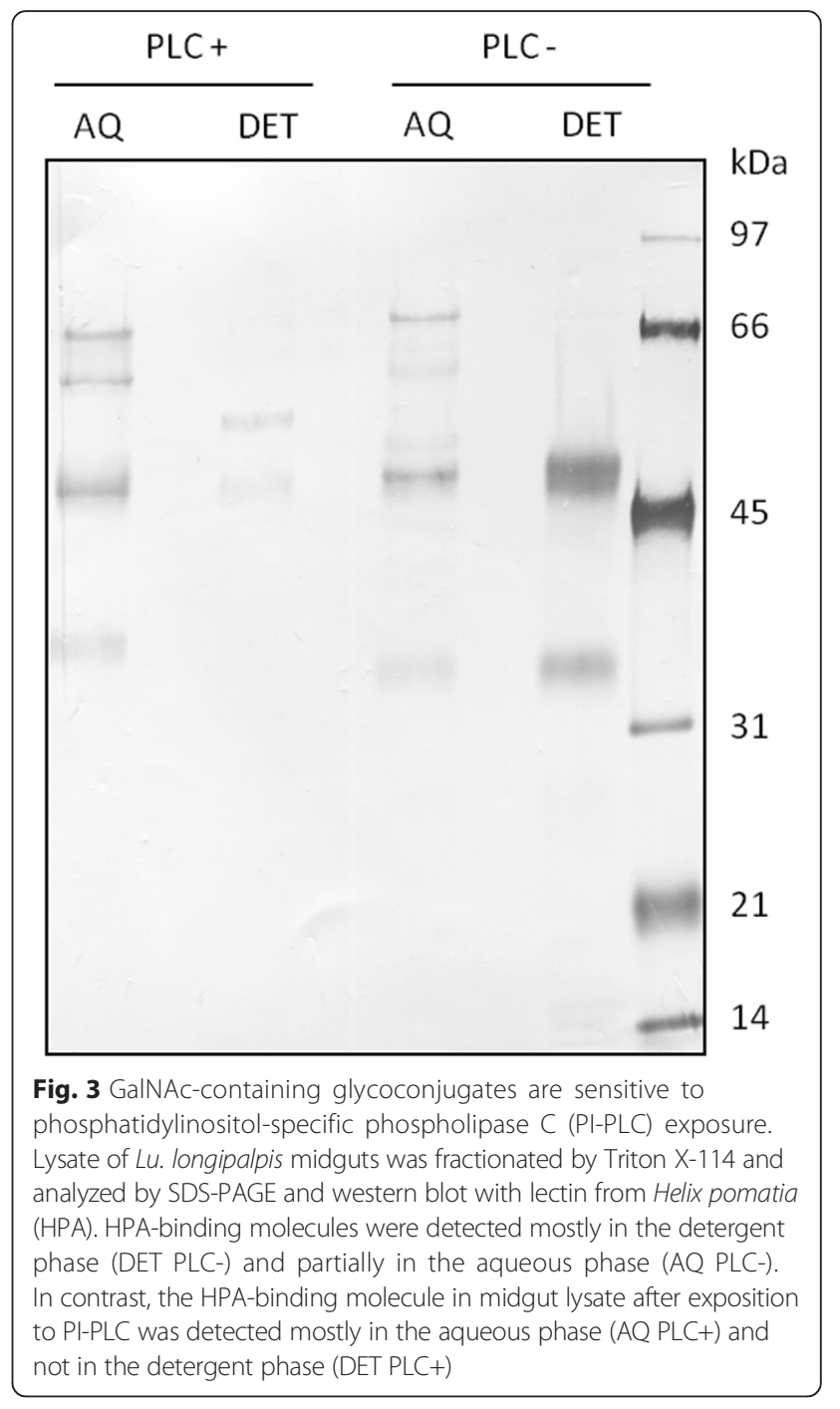

peptides covering $59 \%$ of rLuloG sequence. Rabbit antiserum raised against purified rLuloG was found to strongly recognize the native glycoconjugate from $L u$. longipalpis midguts as well the recombinant molecule. The lectin HPA recognized the same protein band, no reaction was found with preimunne serum (Fig. 5).

To further investigate the role of the Lu. longipalpis glyconconjugate in Leishmania attachment, parasites were incubated with recombinant Lulo protein, followed by anti-rLuloG serum incubation and Alexa-anti rabbit IgG labelling. Results showed that the rLuloG protein specifically bound to the surface of Leishmania, since the whole cell body, including the flagellum was brightly stained by green fluorescent staining (Fig. 6). In contrast, parasites incubated with preimunne serum instead of anti-rLuloG serum were not stained with Alexa-anti rabbit IgG. Lack of staining was observed also in other negative controls: parasites incubated with BSA instead of rLuloG protein, then with anti-rLuloG serum and 


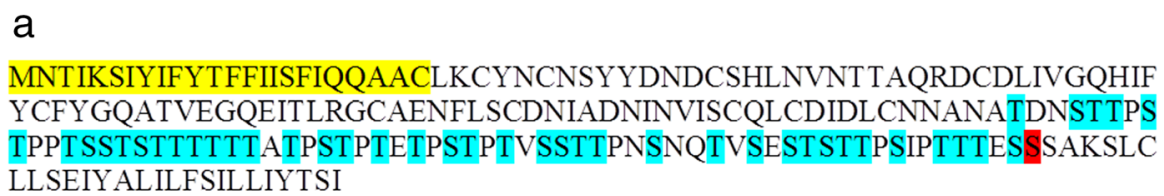

b

NetoGlyc 3.1: predicted $0-g l y c o s y l a t i o n$ sites in gi. 15767443

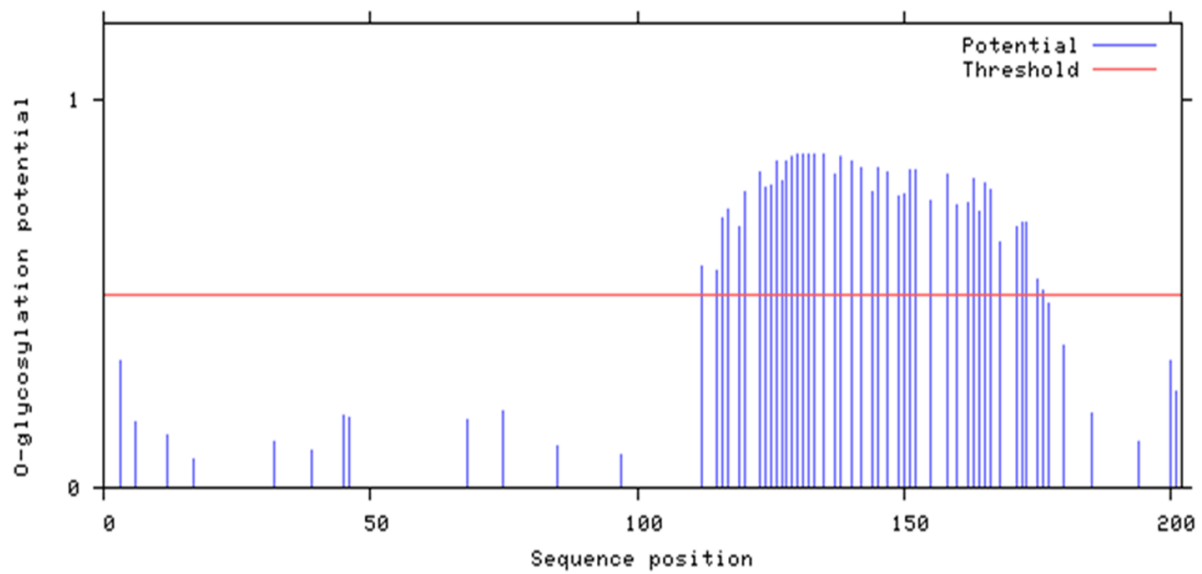

Fig. 4 Lulo protein sequence with C-terminal part rich in potentially O-glycosylated Ser/Thr residues a Highlighted in blue are potentially O-glycosylated Ser/Thr residues (NetOGlyc: http://www.cbs.dtu.dk/services/NetOGlyc/); highlighted in yellow is a signal peptid (http:// www.cbs.dtu.dk/services/SignalP/); site highlighted in red is the GPI-anchor predicted place (http://gpcr2.biocomp.unibo.it/gpipe/index.htm) b Predicted O-glycosylation sites; blue lines going above the treshold line (red) show potential O-glycosylated sites

followed by Alexa staining or parasites incubated with Alexa-anti rabbit IgG only (Fig. 6).

Anti-rLuloG antibodies were used to localize the $L u$. longipalpis mucin-like glycoconjugate on dissected midguts by immunofluorescence. Midguts dissected from blood-fed and defecated Lu. longipalpis were longitudinally opened and incubated with preimmune serum or anti-rLuloG antibodies, followed by incubation with FITC-anti rabbit IgG. Midguts preincubated with preimmune serum were stained mainly nonspecifically on the outer surface where only muscle fibres are visible in the fluorescent microscope. Midguts preincubated with antirLuloG serum revealed strong reaction with the epithelial (inner) midgut surface composed of spherical/round epithelial cells, which are present only in the midgut luminal side [17] (Fig. 7).

\section{Development of $L$. infantum in Lu. longipalpis midgut fed with anti-rLuloG antibodies}

In order to test whether the glycoconjugate from $L u$. longipalpis midguts could be the ligand of the sand fly to which Leishmania parasites attach in vivo, bloodfeeding experiments were performed, where the rabbit serum in blood was reconstituted with anti-rLuloG serum. On day 2 post-infection (PI), prior to defecation of the digested blood meal, there was no significant difference in the number and localization of parasites in the midgut of sand flies fed on blood with anti-rLuloG serum and in blood with normal rabbit serum. Very high infection rates $(100 \%)$ were present in both groups, with heavy infections in $98 \%$ of dissected flies. On day 4 PI, when the blood meal was defecated, females infected with parasites mixed with normal serum retained a high infection rate (almost $100 \%$ ) and high parasite loads, with heavy infection in $83 \%$ of flies. However, females infected with parasites mixed with anti-rLuloG serum showed a decrease in parasite loads, with heavy infection in $78 \%$ of flies (Fig. 8). Although the inhibitory effect of anti-rLuloG antibodies on the parasite presence in the sand fly midgut was not statistically significant, there was a tendency to lower parasite numbers on day 4 . It correlated with the time of Leishmania attachment to the sand fly midgut and subsequent defecation of blood meal remnants which occurs on days 3-5 days PI.

\section{Discussion}

Data reported by Myšková and colleagues [9] suggested that a $45 \mathrm{kDa}$ O-linked glycoprotein on the Lu. longipalpis midgut epithelium may function as a parasite ligand. Here we used a combination of biochemical, molecular and parasitological approaches to characterize the biological properties of this glycoconjugate. We provide evidence that the molecule with an apparent molecular weight $45 \mathrm{kDa}$ on SDS-PAGE corresponds to a putative $19 \mathrm{kDa}$ protein with unknown function detected in a midgut cDNA library of Lu. longipalpis [18]. The 


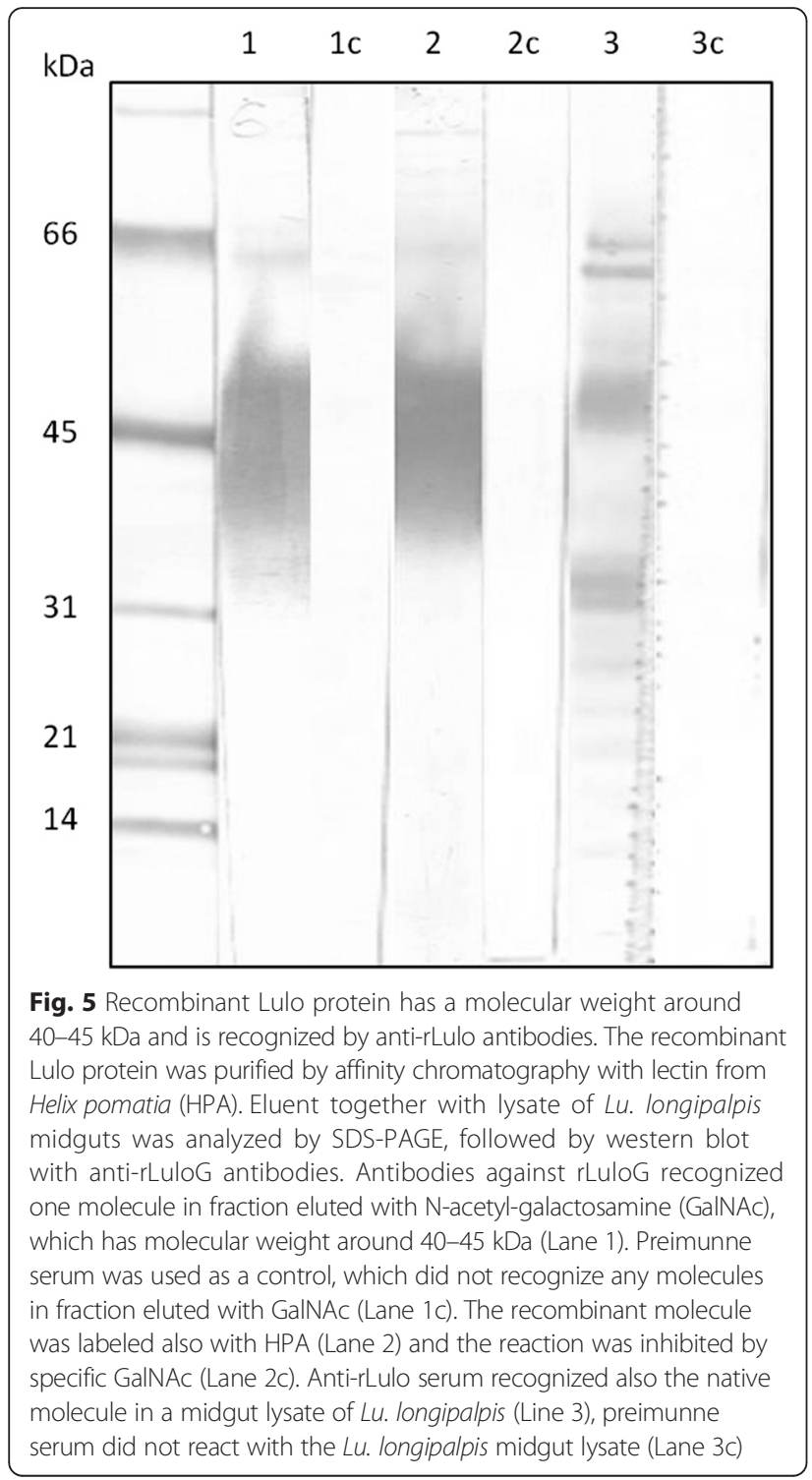

difference between the size of the native protein and predicted protein from cDNA library is assumed to be caused by a high degree of glycosylation. This is in agreement with the numerous predicted glycosylated serine and threonine residues found in the $\mathrm{C}$ - terminal part of the protein sequence, and also corresponds with the smeared appearance of the $45 \mathrm{kDa}$ band on the gels or blots.

O-glycosylation prediction on the entire set of putative proteins identified in the midgut transcriptome of Lutzomyia longipalpis [18] did indeed find LuloG as the most heavily O-glycosylated protein. Beside LuloG, one more putative protein with extensive glycosylation was identified: ABV60335. It is a putatively secreted protein of $16.6 \mathrm{kDa}$ with no other apparent conserved domains. No protein with a similar degree of O-glycosylation (a cluster of more than 40 putatively glycosylated Ser/Thr residues) was identified in the $P$. papatasi midgut transcriptome data [19].

An essential prerequisite for this GalNAc displaying molecule of permissive sand flies to function as a midgut receptor for Leishmania is its abundance and localization on midgut epithelial cells. Figure 7 shows that antibodies against the recombinant LuloG protein recognized round/ spherical cells of dissected $\mathrm{Lu}$. longipalpis midguts, typical of the luminal surface of epithelial midgut cells [17]. On the other hand, preimunne serum recognized mostly musculature, consisting of circular and longitudinal fibres, which is located on the external side of sand fly midgut [20].

Although we could not prove involvement of LuloG in Leishmania attachment by blocking of the native protein on the epithelial surface with anti-rLuloG during sand fly infections, we demonstrated a strong binding ability of rLuloG to whole Leishmania body by in vitro indirect imunofluorescence experiments. This suggests that the receptor for the sand fly glycoconjugate is localized on the entire surface of promastigotes, including the body and flagellum and is accessible to the sand fly midgut ligand. Our data correspond with the observations of Myšková and colleagues [9], where midgut lysates of the permissive sand fly $P$. halepensis bound to Leishmania promastigote surfaces, and this interaction was visualized by FITC labelled HPA, the lectin specific for GalNAc.

We can speculate about the reason for the in vivo blocking attachment results. First, anti-rLuloG antibodies could be proteolytically degraded during blood digestion in the sand fly midgut, which is commonly observed in oral immunotherapy used for mammals. Proteolytic enzymes involved in the degradation of orally administered immunoglobulins in humans include pepsin, trypsin, chymotrypsin, carboxypeptidase and elastase [21]. In P. papatasi and Lu. longipalpis midguts, trypsinlike proteases were described as the most abundant. Secondly, the effectivity of anti-rLuloG antibodies in Leishmania attachment could be limited due to the recombinant protein rLuloG being expressed in soluble form without a GPI anchor, which was used for antibody production. Important roles for GPI anchors in binding of antibodies was demonstrated by numerous studies where removal of the GPI lipid moiety can influence ligand binding properties, most likely due to conformational changes in the protein. Studies showed that antibodies raised against the soluble form of proteins sometimes do not react well with the membrane-bound protein on the living organism, reviewed in [22]. Seeing this unsuccesful inhibiton of Leishmania attachment in vivo, we performed ex vivo inhibition of Leishmania infantum binding to Lutzomyia longipalpis midgut by binding experiments. Details of the method are given by 

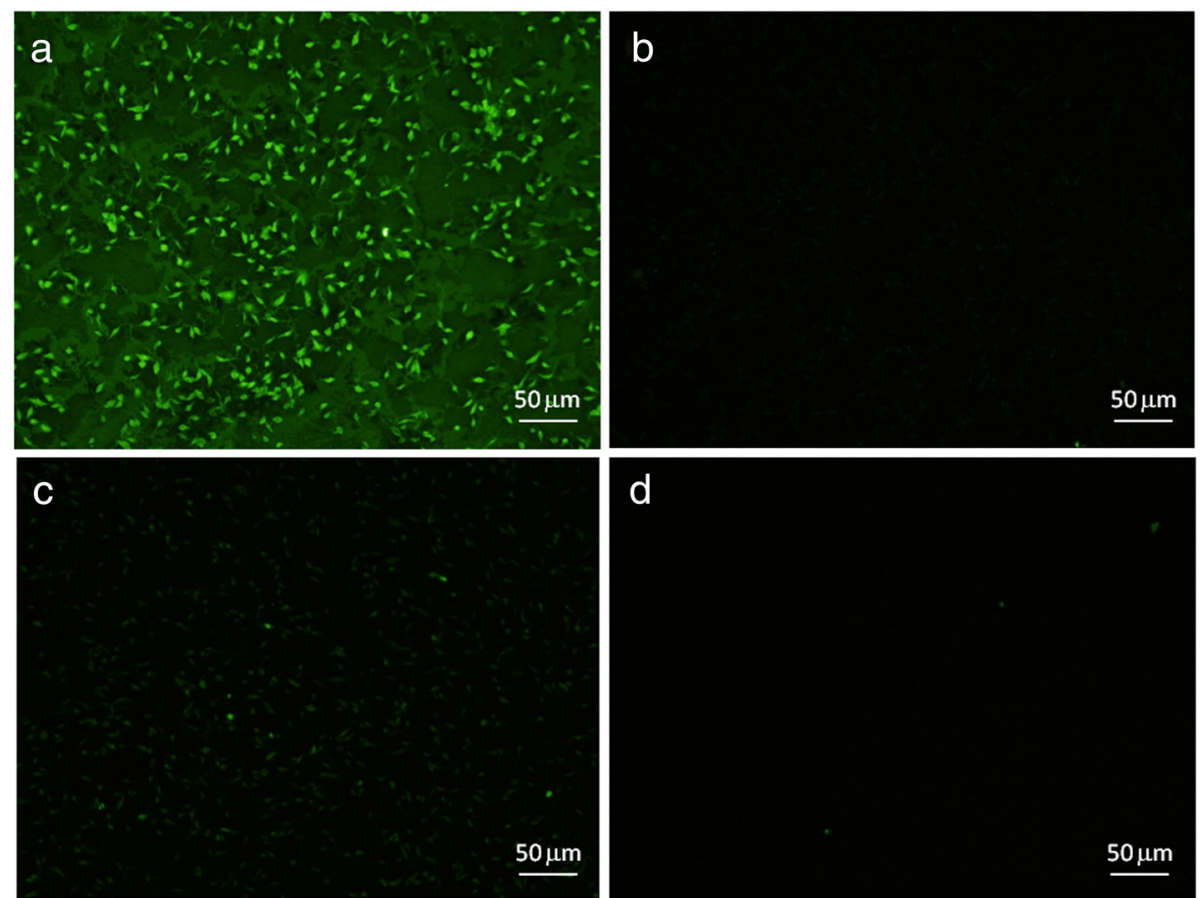

Fig. 6 Binding of recombinant Lulo protein to Leishmania major promastigotes. Promastigotes were fixed on slides and incubated with recombinant Lulo protein, followed by anti-rLulo serum incubation and Alexa-anti rabbit lgG labelling. The entire body, including the flagellum was brightly stained by green fluorescent staining (a). In contrast, parasites incubated with preimunne serum instead of anti-rLulo serum were not stained with Alexa-anti rabbit lgG (b). In the negative controls, parasites incubated with BSA instead of rLulo protein, then with anti-rLulo serum and followed by Alexa staining were stained only weakly (c) and parasites incubated only with Alexa fluor labelling were not stained at all (d)

Wilson and coworkers [23]. Although we did not find significant reduction of attached parasites in the presence of anti rLuloG, again a tendency to lower parasite numbers was observed (data not shown).

Nevertheless, a key question is what kind of parasite molecule could interact with this glycoconjugate. We may speculate that the parasite receptor for the $45 \mathrm{kDa}$ glycoconjugate is a lectin-like molecule with predicted specificity for GalNAc, mimicking the activity of HPA lectin. Lectin activities and heparin-binding activities were demonstrated in Leishmania promastigotes by various authors [24-28]. More recent studies suggest that $65 \mathrm{kDa}$ and $55 \mathrm{kDa}$ heparin-binding proteins from the surface of $L$. braziliensis promastigotes can be involved in parasite adhesion to Lu. longipalpis cells through heparan sulphate bridges [29-31]. These heparin-binding proteins were reported to possess metallo-proteinase like activity [31]. As they were not characterized by mass spectrometry, one cannot exclude the possibility that they are in reality identical with some isoforms of the major surface protease, leishmanolysin/gp 63 (reviewed in [32]).

We predict that the parasite receptor would be expressed at a reasonably high level, to ensure that specific binding to the midgut can occur despite the presence of other high copy surface molecules such as LPG.
Reviewing current knowledge of the promastigote surface there is a variety of candidate molecules. These include leishmanolysin/gp63, PSA/gp46, mPPG, lamininbinding proteins or the already mentioned heparinbinding proteins, and maybe other unidentified proteins. A potential role for gp63 in parasite attachment has been previously proposed [33,34]. These studies showed that the monoxenous trypanosomatids Herpetomonas samuelpessoai and Leptomonas species produce a metallopeptidase that has similar properties to Leishmania gp63 and contributes to parasite adhesion to Aedes aegypti guts or the Aedes albopictus cell line C6/36. These results are consistent with those obtained with Leishmania species; using an ex vivo by binding assay Jecna and colleagues [11] found that the gp63 of L. amazonensis gp63 down-regulated transfectants is functionally important for binding of its promastigotes to the $L u$. longipalpis midgut.

In conclusion, while the L. major-P. papatasi work has established an important paradigm, recent evidence indicates that another mechanism of attachment could exist in permissive vectors. The present study has revealed interesting and novel properties of the " $19 \mathrm{kDa}$ midgut protein" from $\mathrm{Lu}$. longipalpis midguts that can be briefly summarized as follows: (i) it is a mucin-like glycoconjugate with apparent weight of $45 \mathrm{kDa}$; (ii) it is located on 

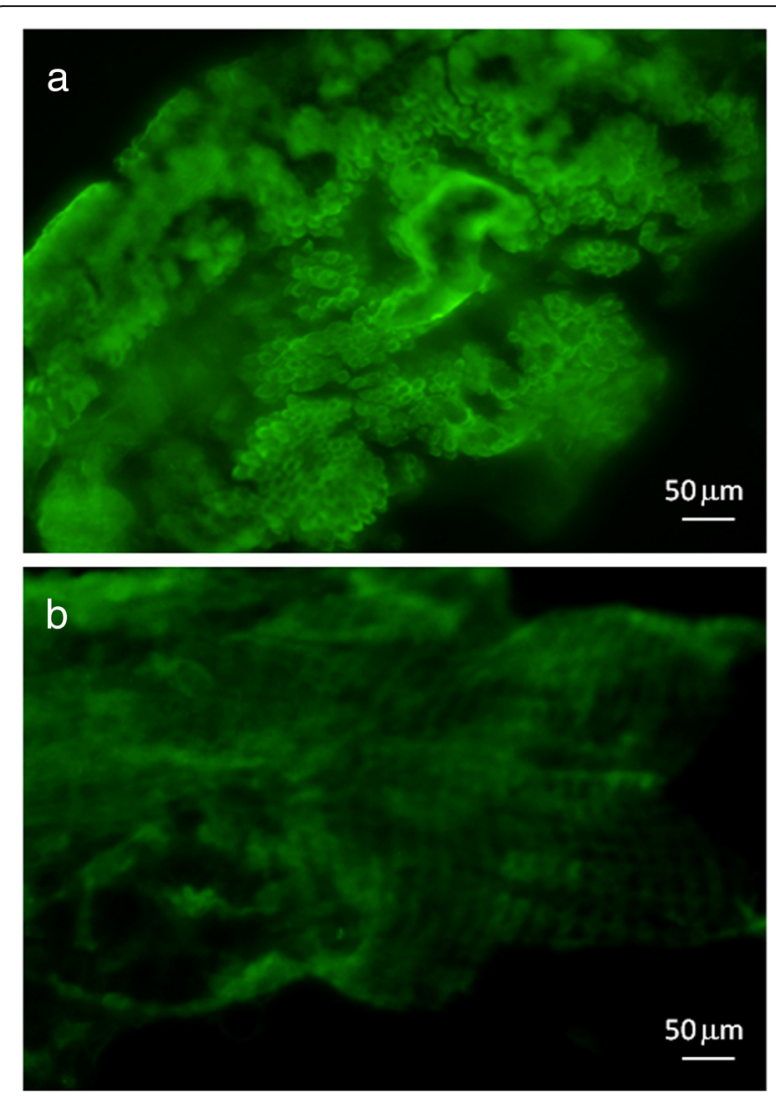

Fig. 7 Binding of anti-rLulo antibodies to Lutzomyia longipalpis midgut epithelium. To investigate the distribution of GalNAc-displaying molecules across the midgut, midguts of Lu. longipalpis were fixed and incubated with either anti-rLulo sera (a) or preimunne sera (b) and labelled with FITC-anti rabbit lgG. Both groups of midguts were stained with similar intensity, but only in guts preincubated with anti-rLulo serum we observed staining of typical spherical/round epithelial cells, which are located on the luminal surface of midgut [17]

the luminal surface of the midgut; and (iii) recombinant form rLuloG is able to bind to the surface of Leishmania cells. Nevertheless, additional studies will be necessary to prove the biological role of the " $19 \mathrm{kDa}$ midgut protein", especially its role as a putative midgut ligand in permissive vectors.

\section{Conclusions}

In this study we characterized a glycoprotein from Lutzomyia longipalpis midgut which has been previously proposed as a molecule participating in Leishmania binding to the epithelium of the permissive sand flies. We identified that this glycoprotein corresponds to a putative $19 \mathrm{kDa}$ protein with unknown function detected in a midgut cDNA library of Lu. longipalpis and named it LuloG. We demonstrate that this mucin-like molecule is GPI-anchored in the membrane of enterocytes and localized it on the luminal side of the midgut. These findings prove accessibility of the mucin for the parasites

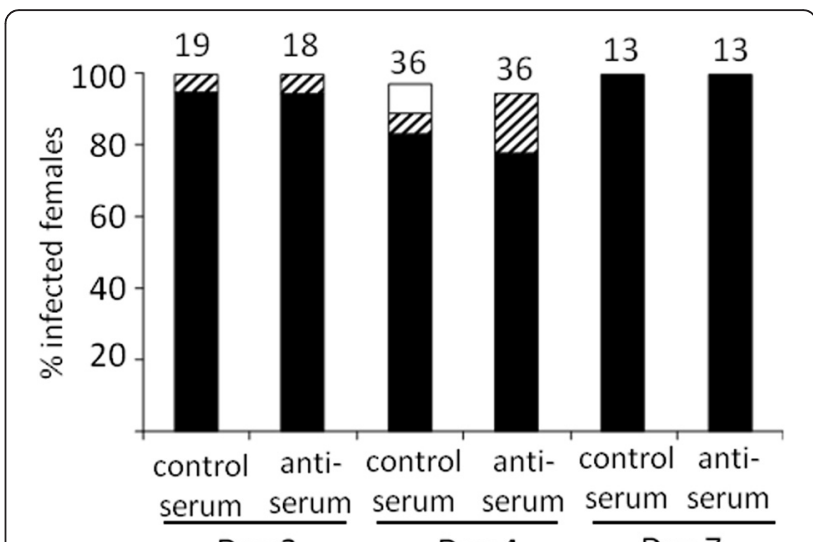

Day 2

Day 4

Day 7

Fig. 8 In vivo inhibition of Leishmania chagasi binding to Lutzomyia longipalpis midgut by anti-rLulo antibodies. Lu. longipalpis females were fed with Leishmania chagasi wild type in blood reconstituted with anti-rLulo serum (anti-serum) or normal rabbit blood (control serum). Day 2 dissections were before defecation, day 4 and 7 dissections were after defecation. Parasite loads were classified into three categories: heavy (more than 1000 parasites per gut; black bars); moderate (100-1000 parasites per gut; striped bars); light (1-100 parasites per gut; white bars). Numbers above the bars indicate the number of dissected females. On day 2 postinfection, both groups of sand flies initially produced heavy or moderate parasite loads in the abdominal midgut. However, after defecation on day 4, intensity of infection was slightly lower in sand flies fed on blood with anti-rLulo serum. In the following days, both groups developed uniformly, with heavy infection in all dissected guts. Data are from two representative experiments

during blood meal digestion. In line with the putative role of mucin in parasite attachment, we indeed showed binding of a recombinant form of the glycoprotein to the surface of Leishmania promastigotes including flagella in vitro. However, antibodies raised against recombinat LuloG were not able to block Leishmania infection in Lu. longipalpis in vivo. In conclusion, our data support strongly the view that LuloG participates in Leishmania attachment to the Lu. longipalpis midgut and thus is important for parasite transmission.

\section{Acknowledgments}

We are grateful to Dr. Jitka Forstová and Dr. Martin Fraiberk and his colleagues who helped us to produce rLuloG in insect cells.

\section{Funding}

The work was supported by Grant 078937 from The Wellcome Trust. The authors gratefully acknowledge the support by the project LO1509 of the Ministry of Education, Youth and Sports of the Czech Republic and by Operational Program Prague - Competitiveness project (CZ.2.16/3.1.00/ 24023) supported by EU.

Availability of data and material

Data supporting the conclusions of this article are included within the article.

\section{Authors' contributions}

Substantial contribution to conception and design: PV, PB; acquisition of data: $A D, L P, J M, P H$; interpretation of data: JM, AD, LP, PH, PV; drafting the article: JM, PV, LP; revising the article for important intellectual content: PV, $\mathrm{PB}, \mathrm{JM}$. All authors read and approved the final version of the manuscript. 


\section{Competing interests}

The authors declare that they have no competing interests.

\section{Consent for publication}

Not applicable.

\section{Ethics approval and consent to participate}

Animals were maintained and handled in the animal facility of Charles University in Prague in accordance with institutional guidelines and Czech legislation (Act Number 246/1992 and 359/2012 coll. on Protection of Animals against Cruelty in present statutes at large), which complies with all relevant uropean Union and international guidelines for experimental animals. The experiments (including sand fly feeding) were approved by the Committee on the Ethics of Animal Experiments of the Charles University in Prague (Permit Number 24/773/08-10001) and were performed under the Certificate of Competency (Registration Number CZU945/05 ext. CZ02573) and the Permission Number 31114/2013-MSMT-13 ext. 24115/ 2014-MZE-17214 of the Ministry of the Environment of the Czech Republic.

\section{Author details}

'Department of Parasitology, Faculty of Science, Charles University, Viničná 7, 12844 Prague 2, Czech Republic. Institute of Microbiology of the ASCR, v.vi.i., Videňská 1083, 14220 Prague 4, Czech Republic. ${ }^{3}$ Division of Biomedical and Life Sciences, Faculty of Health and Medicine, Lancaster University, Lancaster, UK.

Received: 23 March 2016 Accepted: 12 July 2016

\section{Published online: 25 July 2016}

\section{References}

1. Dostálová A, Volf P. Leishmania development in sand flies: parasite-vector interactions overview. Parasit Vectors. 2012;5:276

2. Killick-Kendrick R, Molyneux DH, Ashford RW. Ultrastructural observations on the attachment of Leishmania in the sandfly. Trans R Soc Trop Med Hyg. 1974;68:269-76.

3. Warburg A, Tesh RB, McMahon-Pratt D. Studies on the Attachment of Leishmania flagella to sand fly midgut epithelium. J Protozool. 1989;36:613-7.

4. Pimenta P, Turco S, McConville M, Lawyer P, Perkins P, Sacks D. Stagespecific adhesion of Leishmania promastigotes to the sandfly midgut. Science. 1992:256:1812-5.

5. Pimenta PF, Saraiva EM, Rowton E, Modi GB, Garraway LA, Beverley SM, et al. Evidence that the vectorial competence of phlebotomine sand flies for different species of Leishmania is controlled by structural polymorphisms in the surface lipophosphoglycan. Proc Natl Acad Sci U S A. 1994;91:9155-9.

6. Kamhawi S, Ramalho-Ortigao M, Pham VM, Kumar S, Lawyer PG, Turco SJ, Barillas-Mury C, Sacks DL, Valenzuela JG. A role for insect galectins in parasite survival. Cell. 2004;119:329-41.

7. Dobson DE, Kamhawi S, Lawyer P, Turco SJ, Beverley SM, Sacks DL. Leishmania major Survival in Selective Phlebotomus papatasi Sand fly vector requires a specific SCG-encoded lipophosphoglycan galactosylation pattern. PLoS Pathog. 2010;6:e1001185.

8. Di-Blasi T, Lobo AR, Nascimento LM, Córdova-Rojas JL, Pestana K, Marín-Villa M, et al. The Flagellar protein FLAG1/SMP1 is a candidate for Leishmania -sand fly interaction. Vector-Borne Zoonotic Dis. 2015;15:202-9.

9. Myšková J, Svobodová M, Beverley SM, Volf P. A lipophosphoglycanindependent development of Leishmania in permissive sand flies. Microbes Infect. 2007;9:317-24.

10. Svárovská A, Ant TH, Šeblová V, Jecná L, Beverley SM, Volf P. Leishmania major glycosylation mutants require phosphoglycans (lpg2-) but not lipophosphoglycan (lpg1-) for survival in permissive sand fly vectors. PLoS Negl Trop Dis. 2010;4:e580.

11. Jecná L, Dostálová A, Wilson R, Seblová V, Chang KP, Bates PA, Volf P. The role of surface glycoconjugates in Leishmania midgut attachment examined by competitive binding assays and experimental development in sand flies. Parasitology. 2013;140:1026-32.

12. Volf $P$, Myšková J. Sand flies and Leishmania: specific versus permissive vectors. Trends Parasitol. 2007;23:91-2.

13. Svobodová M, Votypka J, Pecková J, Dvorak V, Nasereddin A, Baneth G, et al. Distinct transmission cycles of Leishmania tropica in 2 adjacent foci, northern Israel. Emerg Infect Dis. 2006;12:1860-8.
14. Volf P, Volfová $V$. Establishment and maintenance of sand fly colonies. J Vector Ecol. 2011;36 Suppl 1:S1-9.

15. Bordier C. Phase separation of integral membrane proteins in Triton X-114. J Biol Chemist. 1981;256:1604-7.

16. Myšková J, Votypka J, Volf P. Leishmania in Sand Flies: Comparison of quantitative polymerase chain reaction with other techniques to determine the intensity of infection. J Med Entomol. 2008;45:133-8.

17. Warburg A. The structure of the female sand fly (Phlebotomus papatasi) alimentary canal. Trans R Soc Trop Med Hyg. 2008;102:161-6.

18. Jochim RC, Teixeira CR, Laughinghouse A, Mu J, Oliveira F, Gomes RB, et al. The midgut transcriptome of Lutzomyia longipalpis: comparative analysis of cDNA libraries from sugar-fed, blood-fed, post-digested and Leishmania infantum chagasi-infected sand flies. BMC Genomics. 2008;9:15.

19. Ramalho-Ortigão M, Jochim RC, Anderson JM, Lawyer PG, Pham VM, Kamhawi S, Valenzuela JG. Exploring the midgut transcriptome of Phlebotomus papatasi: comparative analysis of expression profiles of sugarfed, blood-fed and Leishmania major-infected sandflies. BMC Genomics. 2007:8:300.

20. Secundino NFC, Nacif-Pimenta R, Hajmova M, Volf P, Pimenta PFP. Midgut muscle network in Lutzomyia longipalpis and Phlebotomus duboscai sand flies: spatial organization and structural modification after blood meal. Arthropod Struct Dev. 2005;34:167-78.

21. Reilly RM, Domingo R, Sandhu J. Oral delivery of antibodies. Future pharmacokinetic trends. Clin Pharmacokinet. 1997;32:313-23.

22. Bütikofer $P$, Malherbe $T$, Boschung $M$, Roditi I. GPI-anchored proteins: now you see 'em, now you don't. FASEB J. 2001;15:545-8.

23. Wilson R, Bates MD, Dostalova A, Jecna L, Dillon RJ, Volf P, Bates PA. Stage-Specific Adhesion of Leishmania promastigotes to sand fly midguts assessed using an improved comparative binding assay. PLoS Negl Trop Dis. 2010;4:e816.

24. Hernandez A, Rodriguez N, Stojanovic D, Candelle D. The localization of a lectinlike component on the Leishmania cell surface. Mol Biol Rep. 1986;11:149-53.

25. Mukhopadhyay N, Shome K, Saha A, Hassell J, Glew R. Heparin binds to Leishmania donovani promastigotes and inhibits protein phosphorylation. Biochem J. 1989;264:517-25.

26. Schottelius J. Neoglycoproteins as tools for the detection of carbohydrate-specific receptors on the cell surface of Leishmania. Parasitol Res. 1992;78:309-15.

27. Svobodová M, Bates PA, Volf P. Detection of lectin activity in Leishmania promastigotes and amastigotes. Acta Trop. 1997;68:23-35.

28. Kock N, Gabius H, Schmitz J, Schottelius J. Receptors for carbohydrate ligands including heparin on the cell surface of Leishmania and other trypanosomatids. Trop Med Int Heal. 1997;2:863-74.

29. Azevedo-Pereira RL, Pereira MCS, Oliveria-Junior FOR, Brazil RP, Côrtes LMC, Madeira MF, et al. Heparin binding proteins from Leishmania (Viannia) braziliensis promastigotes. Vet Parasitol. 2007;145:234-9.

30. de Castro Côrtes L, de Souza Pereira M, da Silva F, Pereira BA, de Oliveira Junior $F$, de Araújo Soares $R$, et al. Participation of heparin binding proteins from the surface of Leishmania (Viannia) braziliensis promastigotes in the adhesion of parasites to Lutzomyia longipalpis cells (Lulo) in vitro. Parasit Vectors. 2012;5:142.

31. de Castro Côrtes LM, de Souza Pereira MC, de Oliveira FOR, Corte-Real S, da Silva FS, Pereira BAS, et al. Leishmania (Viannia) braziliensis: insights on subcellular distribution and biochemical properties of heparin-binding proteins. Parasitology. 2012;139:200-7.

32. Yao C, Donelson JE, Wilson ME. The major surface protease (MSP or GP63) of Leishmania sp. Biosynthesis, regulation of expression, and function. Mol Biochem Parasitol. 2003;132:1-16.

33. Pereira FM, Bernardo PS, Dias Junior PFF, Silva BA, Romanos MTV, D'AvilaLevy CM, et al. Differential influence of gp63-like molecules in three distinct Leptomonas species on the adhesion to insect cells. Parasitol Res. 2009;104: 347-53.

34. Pereira FM, Dias FA, Elias CGR, d'Avila-Levy CM, Silva CS, Santos-Mallet JR, Branquinha MH, Santos ALS. Leishmanolysin-like molecules in Herpetomonas samuelpessoai mediate hydrolysis of protein substrates and interaction with insect. Protist. 2010;161:589-602. 